\title{
Do not miss the bridge
}

\author{
Bahaaldin Alsoufi, MD
}

\footnotetext{
From the Division of Cardiothoracic Surgery, Emory University School of Medicine, Children's Healthcare of Atlanta, Atlanta, Ga.

Disclosures: Author has nothing to disclose with regard to commercial support.

Received for publication Feb 26, 2018; accepted for publication Feb 28, 2018; available ahead of print March 28, 2018.

Address for reprints: Bahaaldin Alsoufi, MD, Division of Cardiothoracic Surgery, Emory University School of Medicine, Children's Healthcare of Atlanta, 1405 Clifton Rd NE, Atlanta, GA 30322 (E-mail: balsoufi@ hotmail.com).

J Thorac Cardiovasc Surg 2018;156:1627-8

$0022-5223 / \$ 36.00$

Copyright (C) 2018 by The American Association for Thoracic Surgery

https://doi.org/10.1016/j.jtcvs.2018.02.082
}

A myocardial bridge is a congenital coronary artery anomaly that involves the left anterior descending artery in most cases. It is characterized by the presence of a segment of the coronary artery, the tunneled segment, that takes an intramural course through the myocardium underneath muscular fibers, the muscular bridge. ${ }^{1,2}$ The muscular bridge is not a new entity, having first been described in autopsies by Reyman in 1737, and has been reported to have a prevalence as great as $86 \%$ in autopsy series. ${ }^{1-3}$ Historically, myocardial bridges have been considered benign anatomic variants without hemodynamic or physiologic relevance. Clinical interest has increased in the past 2 decades, however, as a result of (1) emergence of multiple reports associating those bridges with various ischemic myocardial complications, including sudden death, and (2) increased detection of those bridges with advanced imaging techniques, which have also improved our understanding of their pathophysiology causing coronary blood flow compromise because of both highpressure gradients across the tunneled segment, with subsequent decreased coronary flow reserve distally, and plaque formation upstream to the tunneled segment. ${ }^{1,2,4,5}$

In the past 2 decades, a number of small series have described medical, percutaneous, and surgical management of those myocardial bridges. ${ }^{2,6,7}$ Medical therapy with $\beta$-blockers and calcium channel blockers is the mainstay of treatment in patients with symptoms. For patients with refractory symptoms associated with hemodynamically significant disturbances of coronary flow, surgical intervention is warranted. Percutaneous intervention has been performed in those patients, although that has been associated with higher complication rates, including stent fracture and occlusion. Although coronary artery bypass grafting can be performed, this has also been associated with higher incidence of graft occlusion, especially when the thoracic artery has been used, likely because of competitive flow. ${ }^{2}$ Myotomy and unroofing of the myocardial bridges has emerged as a procedure that addresses the underlying pathology in those patients.

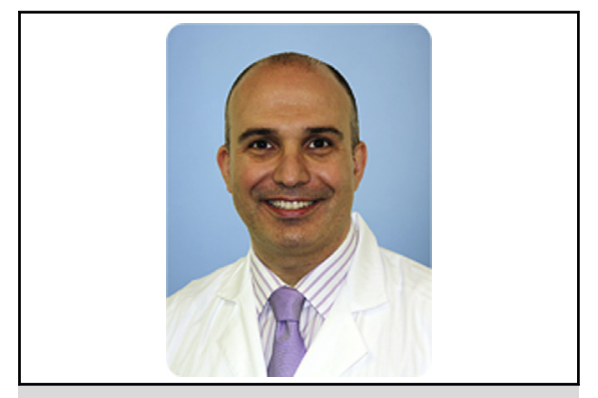

Bahaaldin Alsoufi, MD

Central Message

Surgical unroofing is effective and likely underused procedure in children with symptomatic myocardial bridges.

See Article page 1618 .

Naturally, most series have been reported in adults, and outcomes have been encouraging, with low operative mortality and effective and durable relief of ischemic symptoms. $2,6,7$

In the current issue of the Journal, Maeda and colleagues ${ }^{8}$ from Stanford University report the largest series to date of unroofing of myocardial bridges in the pediatric population. In their series, they operated on 14 children (aged 1120 years) with myocardial bridges between 2012 and 2016. They performed the initial 9 operations on cardiopulmonary bypass, whereas the remaining 5 operations were performed without cardiopulmonary bypass, and with small incisions in the most recent patients. Of interest, 13 of their 14 patients had ischemic symptoms refractory to medical management, and the remaining patient presented with aborted sudden death during exercise. Those patients had significant lesions, with average length of the bridge of $28.2 \pm 16.3 \mathrm{~mm}$ and average halo thickness of $0.59 \pm$ $0.24 \mathrm{~mm}$ on intravenous ultrasonography. In addition, invasive hemodynamic assessment with dobutamine confirmed the physiologic significance of those bridges, with a diastolic fractional flow reserve of $0.59 \pm 0.13$. In their series, there was no significant morbidity or mortality associated with the surgical procedure, and all patients who previously had symptoms (13/13) had improvement in their refractory symptoms and quality of life, as documented by the Seattle Angina Questionnaire. ${ }^{8}$

An interesting finding in the series by Maeda and colleagues $^{8}$ is the prevalence of plaque formation proximal to the tunneled segment even in their pediatric cohort Although the tunneled segment has traditionally been 
reported to stay free of plaques, likely as a result of the systolic compression of those segments, with subsequent improved lymphatic drainage and decreased lipid accumulation in those segments, plaque formation in the artery upstream to the tunneled segment has been frequently reported, especially after the use of intravascular ultrasonography, which helped to detect lesions that were not previously apparent on angiography. ${ }^{1,2,5}$ This is likely due to endothelial injury associated with flow disturbances and wall shear stress at that proximal segment. This plaque formation phenomenon has been well studied and is clearly associated with a more pronounced plaque formation extending more proximally into the coronary ostium. Progression of this plaque is thought to increase with age, as evidenced in the study by Maeda and colleagues. ${ }^{8}$ Their study population was composed of patients between the ages of 11 and 20 years, in whom the plaque burden was almost $19 \%$, lower than that reported in adults $(34 \%))^{4,8}$ Nonetheless, the presence of plaque in this young patient cohort with myocardial bridges is an important and concerning finding that may warrant a more aggressive approach in these cases.

To summarize, the group at Stanford University has operated on 14 children and young adolescents with true symptoms who were treated medically before surgery and continued to have refractory ischemic symptoms. Surgery was safe and effective, and all patients had relief of their symptoms after surgery. This experience from Stanford is both recent (2012-2016) and unique. These procedures are rarely if ever performed at other major pediatric cardiac centers, including Stanford University before 2012. That rarity suggests that physicians are likely missing myocardial bridges as a potential cause of symptoms that may be labeled occasionally as nonspecific. This report is important to shed a light on this pathology in children, especially now that we have excellent advanced imaging modalities at our disposal. As in other coronary artery anomalies, the proper management of patients with incidental findings of myocardial bridges or mild symptoms that are controlled with medical therapy remains elusive. Myocardial bridges, as we have learned recently, are not innocent bystanders and can be associated with acute coronary symptoms, not to mention accelerated and enhanced atherosclerotic disease. It is not known whether patients with asymptomatic myocardial bridges should be medically treated to blunt any exercise or stress response that could cause an acute coronary event, or whether they should be exercise restricted for the same purpose. The hemodynamic and physiologic effects of those bridges likely vary according to the location and length of the tunneled segment, the involvement of branch arteries, the thickness of the overlying muscle fibers, and the association of atherosclerotic disease. Better understanding of the pathophysiology and the natural history of this disease may be warranted to improve our management of those patients and, it is hoped, decrease the incidence of adverse events-notably, sudden death.

This brings to mind a more investigated coronary anomaly, the anomalous origin of the coronary artery from the wrong sinus, which is also associated in some cases with sudden death and continues to raise management controversy, especially in patients with asymptomatic anomalous origin of the right coronary artery from the left sinus. To determine the best management approach of that pathology, the Congenital Heart Surgeons Society is conducting a multi-institutional study examining patients with anomalous origin of the coronary artery from the wrong sinus, including those treated medically and surgically, to determine clinical, physiologic, and anatomic characteristics that warrant surgical intervention. ${ }^{9}$ A similar study may be warranted to understand the clinical impact of myocardial bridges on outcomes and adverse events, and to assess clinical, anatomic, and physiologic variables that might affect the prognosis of those patients. This would not be an easy task to accomplish; however, despite all the medical advances that have been achieve so far, sudden death in children adolescents and young adults continues to be a big burden, even in the most modern societies.

\section{References}

1. Möhlenkamp S, Hort W, Ge J, Erbel R. Update on myocardial bridging. Circulation. 2002;106:2616-22.

2. Corban MT, Hung OY, Eshtehardi P, Rasoul-Arzrumly E, McDaniel M, Mekonnen G, et al. Myocardial bridging: contemporary understanding of pathophysiology with implications for diagnostic and therapeutic strategies. J Am Coll Cardiol. 2014;63:2346-55.

3. Reyman HC. Disertatio de vasis cordis propriis. Bibl Anat. 1737;2:359-79.

4. Erbel R, Rupprecht H-J, Ge J, Gerber T, Görge G, Meyer J. Coronary artery shape and flow changes induced by myocardial bridging. Echocardiography. 1993;10: 71-7.

5. Ishikawa Y, Akasaka Y, Akishima-Fukasawa Y, Iuchi A, Suzuki K, Uno M, et al. Histopathologic profiles of coronary atherosclerosis by myocardial bridge underlying myocardial infarction. Atherosclerosis. 2013;226:118-23.

6. Bockeria LA, Sukhanov SG, Orekhova EN, Shatakhyan MP, Korotayev DA, Sternik L. Results of coronary artery bypass grafting in myocardial bridging of left anterior descending artery. J Card Surg. 2013;28:218-21.

7. Rezayat P, Hassan D, Amirreza S, Susan H. Myocardial bridge. Surgical outcome and midterm follow up. Saudi Med J. 2006;27:1530-3.

8. Maeda K, Schnittger I, Murphy DJ, Tremmel JA, Boyd JH, Peng L, et al. Surgical unroofing of hemodynamically significant myocardial bridges in a pediatric population. J Thorac Cardiovasc Surg. 2018;156:1618-26.

9. Poynter JA, Williams WG, McIntyre S, Brothers JA, Jacobs ML, Congenital Heart Surgeons Society AAOCA Working Group. Anomalous aortic origin of a coronary artery: a report from the Congenital Heart Surgeons Society Registry. World J Pediatr Congenit Heart Surg. 2014;5:22-30. 